PROCEEDINGS OF THE

AMERICAN MATHEMATICAL SOCIETY

Volume 133, Number 6, Pages 1685-1691

S 0002-9939(05)07810-X

Article electronically published on January 13, 2005

\title{
DETERMINING A SOUND-SOFT POLYHEDRAL SCATTERER BY A SINGLE FAR-FIELD MEASUREMENT
}

\author{
GIOVANNI ALESSANDRINI AND LUCA RONDI
}

(Communicated by M. Gregory Forest)

\begin{abstract}
We prove that a sound-soft polyhedral scatterer is uniquely determined by the far-field pattern corresponding to an incident plane wave at one given wavenumber and one given incident direction.
\end{abstract}

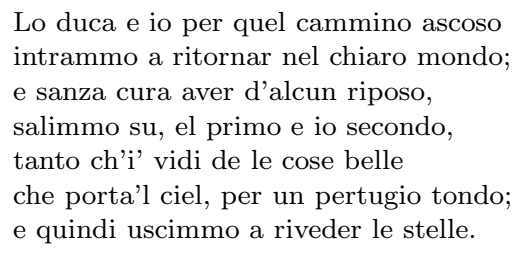

Dante, Inferno, C.XXXIV, 133-139.

\section{INTRODUCTION}

We consider the acoustic scattering problem with a sound-soft obstacle $D$. For simplicity of exposition, let us assume here that $D$ is a bounded solid in $\mathbb{R}^{N}, N \geq 2$, that is that $D$ is a connected compact set which coincides with the closure of its interior. We shall denote by $G$ the exterior of $D$,

$$
G=\mathbb{R}^{N} \backslash D,
$$

and we shall assume throughout that it is connected.

Let $\omega \in \mathbb{S}^{N-1}$ and let $k>0$ be fixed. Let $u$ be the complex valued solution to

$$
\begin{cases}\Delta u+k^{2} u=0 & \text { in } G, \\ u(x)=u^{s}(x)+\mathrm{e}^{\mathrm{i} k \omega \cdot x} & x \in G, \\ u=0 & \text { on } \partial G, \\ \lim _{r \rightarrow \infty} r^{(N-1) / 2}\left(\frac{\partial u^{s}}{\partial r}-\mathrm{i} k u^{s}\right)=0 & r=\|x\| .\end{cases}
$$

It is well known that the asymptotic behavior at infinity of the so-called scattered field $u^{s}(x)=u(x)-\mathrm{e}^{\mathrm{i} k \omega \cdot x}$ is governed by the formula

$$
u^{s}(x)=\frac{\mathrm{e}^{\mathrm{i} k\|x\|}}{\|x\|^{(N-1) / 2}}\left\{u_{\infty}(\hat{x})+O\left(\frac{1}{\|x\|}\right)\right\},
$$

Received by the editors January 22, 2004.

2000 Mathematics Subject Classification. Primary 35R30; Secondary 35P25.

Key words and phrases. Inverse acoustic scattering, polyhedra, uniqueness, reflection principle. This work was supported in part by MIUR under grant no. 2002013279.

(C)2005 American Mathematical Society Reverts to public domain 28 years from publication 
as $\|x\|$ goes to $\infty$, uniformly in all directions $\hat{x}=x /\|x\| \in \mathbb{S}^{N-1}$. The function $u_{\infty}$, which is defined on $\mathbb{S}^{N-1}$, is called the far-field pattern of $u^{s}$; see for instance [2]. In this paper we prove that if $N=2$ and $D$ is a polygon, or if $N=3$ and $D$ is a polyhedron, then it is uniquely determined by the far-field pattern $u_{\infty}$ for one wavenumber $k$ and one incident direction $\omega$; see Theorem 2.2 below. Indeed, we prove this result independently of the dimension $N \geq 2$, and for this reason it is convenient to express our assumption on $D$ by prescribing that it is an $N$ dimensional polyhedron, that is, a solid whose boundary is contained in the union of finitely many $(N-1)$-dimensional hyperplanes (more precisely, we should say a polytope (see for instance [3]), but for the sake of simplicity we prefer to stick to the 3-dimensional terminology). In fact, our result applies to a wider class of scatterers $D$, which need not to be solids, nor connected, but whose boundary is the finite union of the closures of open subsets of $(N-1)$-dimensional hyperplanes. See Section 2 below for a complete formulation.

We wish to mention here that in '94 C. Liu and A. Nachman [4] proved, among various results, that, for $N \geq 2, u_{\infty}$ uniquely determines the convex hull of a polyhedral obstacle $D$. Their arguments involve a scattering theory analogue of a classical theorem of Polya on entire functions and the reflection principle for solutions of the Helmholtz equation across a flat boundary. In that preprint, they also presented some arguments towards a proof of the unique determination of a general polyhedral obstacle.

More recently, J. Cheng and M. Yamamoto [1], for the case $N=2$, proved that the far-field pattern uniquely determines a polygonal obstacle $D$, provided $D$ satisfies an additional geometrical condition, which, roughly speaking, is expressed in terms of the absence of trapped rays in its exterior $G$. The method of proof in [1] is mainly based on the use of the reflection principle and on the study of the behavior of the nodal line $\{u=0\}$ of the solution $u$ to (1.2) near the boundary $\partial G$.

Also in this paper we make use of such a reflection argument, but, rather than examining the boundary behavior of the nodal set we investigate the structure of the nodal set of $u$ in the interior of $G$. In this respect, the main tool is summarized in the fact that if $D$ is a polyhedron, then the nodal set of $u$ in $G$ does not contain any open portion of an $(N-1)$-dimensional hyperplane; see Theorem 2.4 .

In Section 2 we set up our main hypotheses on the obstacle, we state the main results (Theorem 2.2 and Theorem 2.4 and prove Theorem 2.2

In Section 3 we prove Theorem 2.4 The proof is preceded by a sequence of propositions and auxiliary lemmas regarding the study of the nodal sets of real-valued solutions to the Helmholtz equation (see Proposition 3.2) and the construction of a suitable path in $G$ (cammino ascoso = hidden path) which connects a point in $\partial D$ to infinity, avoiding the singular points in the nodal set of $u$ and intersecting the nodal set orthogonally, Proposition 3.6.

\section{The Uniqueness Result}

Definition 2.1. Let us define a cell as the closure of an open subset of an $(N-1)$ dimensional hyperplane. We shall say that $D$ is a polyhedral scatterer if it is a compact subset of $\mathbb{R}^{N}$, such that

(i) the exterior $G=\mathbb{R}^{N} \backslash D$ is connected,

(ii) the boundary of $G$ is given by the finite union of cells $C_{j}$. 
Let us observe that an equivalent condition to (ii) is that $D$ has the form

$$
D=\left(\bigcup_{i=1}^{m} P_{i}\right) \cup\left(\bigcup_{j=1}^{n} S_{j}\right),
$$

where each $P_{i}$ is a polyhedron and each $S_{j}$ is a cell; thus we are allowing the simultaneous presence of solid obstacles and of crack-type scatterers. Note also that, by this definition, a cell need not be an $(N-1)$-dimensional polyhedron.

We also recall that for any compact set $D$ a weak solution $u \in W_{l o c}^{1,2}(G)$ to (1.2) exists and is unique; see for instance [5. As is well known, $u$ is analytic in $G$, but, of course, due to the possible irregularity of the boundary of $G$, the Dirichlet boundary condition in (1.2) is, in general, satisfied in the weak sense only. On the other hand, one can see that, if $x^{0} \in \partial G$ is an interior point of one of the cells forming $\partial G$, then it is a regular point for the Dirichlet problem in $G$, hence $u$ is continuous up to $x^{0}$ and $u\left(x^{0}\right)=0$.

Theorem 2.2. Let us fix $\omega \in \mathbb{S}^{N-1}$ and $k>0$. A polyhedral scatterer $D$ is uniquely determined by the far-field pattern $u_{\infty}$.

A proof of Theorem 2.2 will be obtained as a consequence of Theorem 2.4 below; the following definitions will be needed.

Definition 2.3. Let us denote by $\mathcal{N}_{u}$ the nodal set of $u$ in $G$, that is,

$$
\mathcal{N}_{u}=\{x \in G: u(x)=0\} .
$$

We shall say that $x \in \mathcal{N}_{u}$ is a flat point if there exist a hyperplane $\Pi$ through $x$ and a positive number $r$ such that $\Pi \cap B_{r}(x) \subset \mathcal{N}_{u}$.

Theorem 2.4. Let $D$ be a polyhedral scatterer. Then $\mathcal{N}_{u}$ cannot contain any flat point.

We postpone the proof of this result to Section 3 and we conclude the proof of Theorem [2.2.

Proof of Theorem 2.2. Let $D$ and $D^{\prime}$ be two polyhedral scatterers and let $u^{\prime}$ be the solution to (1.2) when $D$ is replaced with $D^{\prime}$. Let us assume that for a given $\omega \in \mathbb{S}^{N-1}$ and $k>0, u_{\infty}=u_{\infty}^{\prime}$. We denote with $\widetilde{G}$ the connected component of $\mathbb{R}^{N} \backslash\left(D \cup D^{\prime}\right)$ which contains the exterior of a sufficiently large ball. By Rellich's Lemma (see for instance [2, Lemma 2.11]) and unique continuation we infer that $u=u^{\prime}$ over $\widetilde{G}$.

First, we notice that if $\partial \widetilde{G} \subset D \cap D^{\prime}$, then $D=D^{\prime}=\mathbb{R}^{N} \backslash \widetilde{G}$. This is due to the fact that both $G$ and $G^{\prime}=\mathbb{R}^{N} \backslash D^{\prime}$ are connected.

Let us assume, by contradiction, that $D$ is different from $D^{\prime}$. Then, without loss of generality, we can assume that there exists a point $x^{\prime} \in\left(\partial G^{\prime} \backslash D\right) \cap \partial \widetilde{G}$. We can also assume that $x^{\prime}$ belongs to the interior of one of the cells composing $\partial G^{\prime}$, and therefore that there exist a hyperplane $\Pi^{\prime}$ and $r>0$ such that $x^{\prime} \in$ $S^{\prime}=\Pi^{\prime} \cap B_{r}\left(x^{\prime}\right) \subset\left(\partial G^{\prime} \backslash D\right) \cap \partial \widetilde{G}$. Since $u=u^{\prime}$ in $\widetilde{G}$, by continuity we have that $u=u^{\prime}=0$ on $S^{\prime}$, hence $S^{\prime}$ is contained into the nodal set of $u$, that is, $S^{\prime} \subset \mathcal{N}_{u}$, and, consequently, $x^{\prime}$ is a flat point for $\mathcal{N}_{u}$. This contradicts Theorem 2.4 . 


\section{The hidden path AND THE PROOF OF THEOREM 2.4}

We start with a well-known property of the nodal set of $u$.

Lemma 3.1. The nodal set $\mathcal{N}_{u}$ is bounded.

Proof. By (1.3), we have that the scattered field $u^{s}(x)$ tends to zero, as $\|x\|$ tends to infinity, uniformly for all directions $\hat{x}=x /\|x\| \in \mathbb{S}^{N-1}$. Then the lemma immediately follows by observing that $|u(x)|=\left|u^{s}(x)+\mathrm{e}^{\mathrm{i} k \omega \cdot x}\right| \rightarrow 1$ uniformly as $\|x\| \rightarrow \infty$.

Next we discuss some properties of the nodal set of real-valued solutions to the Helmholtz equation. Let $v$ be a nontrivial real-valued solution to

$$
\Delta v+k^{2} v=0 \text { in } G
$$

in a connected open set $G$. We denote the nodal set of $v$ as

$$
\mathcal{N}_{v}=\{x \in G: v(x)=0\}
$$

and we let $\mathcal{C}_{v}$ be the set of nodal critical points, that is,

$$
\mathcal{C}_{v}=\{x \in G: v(x)=0 \text { and } \nabla v(x)=0\} .
$$

We say that $\Sigma \subset \mathcal{N}_{v}$ is a regular portion of $\mathcal{N}_{v}$ if it is an analytic open and connected hypersurface contained in $\mathcal{N}_{v} \backslash \mathcal{C}_{v}$. Let us denote by $A_{1}, A_{2}, \ldots, A_{n}, \ldots$ the nodal domains of $v$, that is, the connected components of the open set $\{x \in G: v(x) \neq$ $0\}=G \backslash \mathcal{N}_{v}$.

Proposition 3.2. We can order the nodal domains $A_{1}, A_{2}, \ldots, A_{n}, \ldots$ in such a way that for any $j \geq 2$ there exist $i, 1 \leq i<j$, and a regular portion $\Sigma_{j}$ of $\mathcal{N}_{v}$ such that

$$
\Sigma_{j} \subset \partial A_{i} \cap \partial A_{j} .
$$

We subdivide the main steps of the proof of this proposition in the next two lemmas.

Lemma 3.3. Let $A_{1}, \ldots, A_{n}$ be nodal domains and let $A=\overline{A_{1} \cup \ldots \cup A_{n}}$. If $x \in$ $\partial A \cap G$, then for any $r>0$ there exists $y \in\left(B_{r}(x) \cap G\right) \backslash \bar{A}$.

Proof. We can assume, without loss of generality, that $r>0$ is such that $B_{r}(x) \subset G$. Then, let us assume, by contradiction, that we have $B_{r}(x) \subset \bar{A}$. Then we infer that $x \in \stackrel{\circ}{\bar{A}}=A$ and this contradicts the fact that $x \in \partial A$.

Lemma 3.4. Let $A_{1}, \ldots, A_{n}$ be nodal domains and let $A=\overline{A_{1} \cup \ldots \cup A_{n}}$. If $x \in$ $\partial A \cap G$, then for any $r>0$ there exists $y \in B_{r}(x) \cap \partial A \cap G$ such that $\nabla v(y) \neq 0$.

Proof. Note that, if we a-priori knew that $\partial A$ were smooth in a small neighborhood of $x$, then the thesis would be a straightforward consequence of Holmgren's Theorem. Lacking such an a-priori smoothness, we proceed as follows. We can assume, without loss of generality, that $r>0$ is such that $B_{r}(x) \subset G$. Assume, by contradiction, that $\nabla v \equiv 0$ on $B_{r}(x) \cap \partial A$ and set $w=v$ in $B_{r}(x) \cap A, w=0$ in $B_{r}(x) \backslash A$. One can easily verify that $w \in W^{2, \infty}\left(B_{r}(x)\right)$ and also that $w$ is a strong solution to the Helmholtz equation in $B_{r}(x)$. Now, by Lemma 3.3 $w \equiv 0$ on an open subset of $B_{r}(x)$ and hence by unique continuation $w \equiv 0$ in $B_{r}(x)$ which is impossible. 
Proof of Proposition 3.2. We proceed by induction. We choose $A_{1}$ arbitrarily.

Let us assume that we have ordered $A_{1}, \ldots, A_{n}$ in such a way that there exist $\Sigma_{2}, \ldots, \Sigma_{n}$ regular portions of $\mathcal{N}_{v}$ such that (3.2) holds for any $j=2, \ldots, n$ and for some $i<j$.

Let $A=\overline{A_{1} \cup \ldots \cup A_{n}}$. If $A=G$, then we are done. Otherwise, since $G$ is connected, we can find $x \in \partial A \cap G$. We apply Lemma 3.4 and we fix, for $r>0$ small enough, a point $y \in B_{r}(x) \cap \partial A \cap G$ such that $\nabla v(y) \neq 0$. There exists a positive $r_{1}$ such that $B_{r_{1}}(y) \cap \partial A$ is a regular portion of $\mathcal{N}_{v}$ and there exist exactly two nodal domains, $\tilde{A}_{1} \subset A$ and $\tilde{A}_{2}$ with $\tilde{A}_{2} \cap A=\emptyset$, whose intersections with $B_{r_{1}}(y)$ are not empty. It is clear that $\tilde{A}_{1}$ coincides with $A_{i}$ for some $i=1, \ldots, n$, and if we pick $A_{n+1}=\tilde{A}_{2}$ and choose $\Sigma_{n+1}=B_{r_{1}}(y) \cap \mathcal{N}_{v}$, then (3.2) holds for $j=n+1$, also.

We now show that we are able to connect points of $G \backslash \mathcal{N}_{v}$ with suitable regular curves contained in $G$ which avoid the nodal critical points of $v$. Here and in the sequel we shall say that a curve $\gamma=\gamma(t)$ is regular if it is $C^{1}$-smooth and $\frac{\mathrm{d}}{\mathrm{d} t} \gamma(t) \neq 0$ for every $t$.

Proposition 3.5. Let $x_{1}$ and $y_{1}$ belong to $G \backslash \mathcal{N}_{v}$. Then there exists a regular curve $\gamma$ contained in $G$ and connecting $x_{1}$ with $y_{1}$ such that the following conditions are satisfied:

$$
\begin{gathered}
\gamma \cap \mathcal{C}_{v}=\emptyset, \\
\text { if } x \in \mathcal{N}_{v} \cap \gamma \text {, then } \gamma \text { intersects } \mathcal{N}_{v} \text { at x orthogonally. }
\end{gathered}
$$

Proof. We order the nodal domains $A_{1}, A_{2}, \ldots, A_{n}, \ldots$ according to Proposition 3.2 Without loss of generality, we can assume that $x_{1} \in A_{1}$ and $y_{1} \in A_{i}$ for some $i>1$. By Proposition 3.2 we can find $i_{l}$, with $l=1, \ldots, n$, such that $i_{1}=1, i_{n}=i$, and, for any $l=2, \ldots, n, i_{l-1}<i_{l}$ and there exists a regular portion of $\mathcal{N}_{v}, \Sigma_{i_{l}}$, such that $\Sigma_{i_{l}} \subset \partial A_{i_{l-1}} \cap \partial A_{i_{l}}$.

Let $\sigma_{l}$ be a line segment crossing $\Sigma_{i_{l}}$ orthogonally and let it be small enough such that $\sigma_{l} \subset A_{i_{l-1}} \cup \Sigma_{i_{l}} \cup A_{i_{l}}$. Let $y_{l}^{-} \in A_{i_{l-1}}, y_{l}^{+} \in A_{i_{l}}$ be the endpoints of $\sigma_{l}$. Let $\beta_{1}$ be a regular path within $A_{1}$ which joins $x_{1}$ to $y_{2}^{-}$and has a $C^{1}$-smooth junction with $\sigma_{2}$ at $y_{2}^{-}$. For every $l=2, \ldots, n-1$, let $\beta_{l}$ be a regular path within $A_{i_{l}}$ which joins $y_{l}^{+}$to $y_{l+1}^{-}$and has $C^{1}$-smooth junctions with the segments $\sigma_{l}$ and $\sigma_{l+1}$, at the points $y_{l}^{+}, y_{l+1}^{-}$, respectively. Let $\beta_{n}$ be a regular path within $A_{i_{n}}$ which joins $y_{n}^{+}$to $y_{1}$ and has a $C^{1}$-smooth junction with $\sigma_{n}$ at $y_{n}^{+}$. We form the curve $\gamma$ by attaching consecutively the curves $\beta_{1}, \sigma_{1}, \beta_{2}, \sigma_{2}, \ldots$ up to $\beta_{n}$.

We have what is needed to build up our hidden path. From now on we consider $G=\mathbb{R}^{N} \backslash D$ and $v=\Re u$. Note that $\mathcal{N}_{u} \subset \mathcal{N}_{v}$.

Proposition 3.6. Let $x_{1} \in \partial G$ be such that $x_{1}$ belongs to the interior of one of the cells forming $\partial G$ and $\frac{\partial v}{\partial \nu}\left(x_{1}\right) \neq 0, \nu$ being the unit normal to $\partial G$ at $x_{1}$, pointing to the interior of $G$. Let $y_{1} \in \mathcal{N}_{u} \backslash \mathcal{C}_{v}$ be fixed.

Then there exists a regular curve $\gamma:[0,+\infty) \mapsto \mathbb{R}^{N}$, such that the following conditions are satisfied:

(1) $\gamma(0)=x_{1}$;

(2) $\gamma(t) \in G$ for every $t>0$;

(3) there exists $t_{1}$ such that $\gamma\left(t_{1}\right)=y_{1}$; 
(4) $\lim _{t \rightarrow+\infty}\|\gamma(t)\|=+\infty$;

(5) if, for some $t, \gamma(t) \in \mathcal{N}_{u}$, then $\gamma(t) \notin \mathcal{C}_{v}$ and $\gamma$ intersects $\mathcal{N}_{v}$ at $x=\gamma(t)$ orthogonally.

Proof. Let $A_{1}$ be the nodal domain of $v$ such that $x_{1} \in \partial A_{1}$ and let $\eta_{1}$ be a line segment in $A_{1}$ having $x_{1}$ as an endpoint and which is orthogonal to $\partial G$ there. Let $x_{1}^{\prime} \in A_{1}$ be the other endpoint of $\eta_{1}$. Let $\eta_{2}$ be a line segment crossing $\mathcal{N}_{v}$ orthogonally at the point $y_{1}$. Let it be small enough so that $v$ is strictly monotone on $\eta_{2}$. Let $y_{1}^{\prime}, y_{1}^{\prime \prime}$ be the endpoints of $\eta_{2}$. By Proposition 3.5. we can find a regular curve $\gamma_{1}$ joining $x_{1}^{\prime}$ to $y_{1}^{\prime}$ and satisfying conditions (3.3), (3.4). We can also choose $\gamma_{1}$ in such a way that it has $C^{1}$-smooth junctions with the segments $\eta_{1}, \eta_{2}$ at its endpoints. Let $R>0$ be large enough so that $\mathcal{N}_{u} \subset B_{R}(0)$ and let us fix $z_{1},\left|z_{1}\right|>R$. Again by Proposition [3.5 we can find a regular curve $\gamma_{2}$ joining $y_{1}^{\prime \prime}$ to $z_{1}$ and satisfying conditions (3.3), (3.4) and also such that it has a $C^{1}$-smooth junction with $\eta_{2}$ at the point $y_{1}^{\prime \prime}$. Next let us fix a regular path $\gamma_{3}$ in $\mathbb{R}^{N} \backslash B_{R}(0)$ joining $z_{1}$ to $\infty$ having a $C^{1}$-smooth junction with $\gamma_{2}$ at $z_{1}$. The resulting path $\gamma$ is obtained by attaching the paths $\eta_{1}, \gamma_{1}, \eta_{2}, \gamma_{2}, \gamma_{3}$.

Lemma 3.7. Let the assumptions of Proposition 3.6 be satisfied and let $\gamma$ be the path constructed there. If $y^{\prime}=\gamma\left(t^{\prime}\right) \in \mathcal{N}_{u}$ is a flat point, then there exists $t^{\prime \prime}>t^{\prime}$ such that $y^{\prime \prime}=\gamma\left(t^{\prime \prime}\right) \in \mathcal{N}_{u}$ is a flat point.

Proof. Let $\Pi^{\prime}$ be the plane through $y^{\prime}$ and let $r>0$ be such that $S^{\prime}=\Pi^{\prime} \cap B_{r}\left(y^{\prime}\right) \subset$ $\mathcal{N}_{u}$.

Let $\widetilde{S}^{\prime}$ be the connected component of $\Pi^{\prime} \backslash D$ containing $y^{\prime}$. We have that, by analytic continuation, $u$ is identically zero on $\widetilde{S}^{\prime}$. Therefore, we can immediately notice that, by Lemma $3.1 \widetilde{S}^{\prime}$ is bounded.

Let $\epsilon>0$ be small enough so that $v(\gamma(t))$ is strictly monotone for $t^{\prime}-\epsilon \leq t \leq t^{\prime}+\epsilon$, and let us set $y^{-}=\gamma\left(t^{\prime}-\epsilon\right), y^{+}=\gamma\left(t^{\prime}+\epsilon\right)$.

Let $G^{+}$be the connected component of $G \backslash \widetilde{S}^{\prime}$ containing $y^{+}$and let $G^{-}$be the connected component of $G \backslash \widetilde{S}^{\prime}$ containing $y^{-}$. Let us remark that it may happen that $G^{+}=G^{-}$.

Let us denote with $R$ the reflection in $\Pi^{\prime}$. We call $E^{+}$the connected component of $G^{+} \cap R\left(G^{-}\right)$containing $y^{+}$and $E^{-}$the connected component of $G^{-} \cap R\left(G^{+}\right)$ containing $y^{-}$. We observe that $E^{-}=R\left(E^{+}\right)$and we set $E=E^{+} \cup E^{-} \cup \widetilde{S}^{\prime}$.

We have that $E$ is a connected open set and, by construction, the boundary of $E$ is composed by cells, more precisely by subsets of the cells of $\partial G$ and of $R(\partial G)$. Furthermore, in $E$ we have that $u=-R u$ where $R u(x)=u(R(x))$. In fact, $u+R u$ is a solution of the Helmholtz equation in $E$ with zero Cauchy data on $\widetilde{S}^{\prime}$.

In other words, $u$ is odd symmetric in $E$, with respect to the plane $\Pi^{\prime}$. Hence, we infer that $u=0$ on $E \cap \Pi^{\prime}$ and, moreover, $u$ is continuous up to the interior of each cell forming $\partial E$ and $u=0$ there. Furthermore, since $u$ is continuous in $G$, we have that $u=0$ in all of $\partial E \cap G$. That is, $\partial E \cap G \subset \mathcal{N}_{u}$.

Let us exclude now the case that $E$ is unbounded. In fact, $\partial E$ is bounded and, if $E$ were unbounded, then $E$ would contain $\mathbb{R}^{N} \backslash B_{\rho}(0)$, for some sufficiently large $\rho>0$. Then $u=0$ on $\Pi^{\prime} \backslash B_{\rho}(0)$ and this contradicts Lemma 3.1.

Thus $E$ is a bounded open set containing $y^{\prime}$. Since $\gamma$ is not bounded, there exists $t^{\prime \prime}>t^{\prime}$ such that $\gamma\left(t^{\prime \prime}\right) \in \partial E \cap G$. We have that $y^{\prime \prime}=\gamma\left(t^{\prime \prime}\right) \in \mathcal{N}_{u}$ and, by the properties of $\gamma$, it is not a critical point of $v$. Let $C$ be a cell of $\partial E$ such that $y^{\prime \prime} \in C$ and let $\Pi^{\prime \prime}$ be the hyperplane containing $C$. Let $r>0$ be such that $B_{r}\left(y^{\prime \prime}\right) \subset G$. 
We have that $u=0$ on $C \cap B_{r}\left(y^{\prime \prime}\right)$ and hence, by analytic continuation, $u=0$ on $\Pi^{\prime \prime} \cap B_{r}\left(y^{\prime \prime}\right)$; therefore $\Pi^{\prime \prime} \cap B_{r}\left(y^{\prime \prime}\right) \subset \mathcal{N}_{u}$.

Proof of Theorem 2.4. Let us assume, by contradiction, that $y_{1} \in \mathcal{N}_{u}$ is a flat point. Let $\Pi_{1}$ be the plane through $y_{1}$ and $r>0$ such that $S_{1}=\Pi_{1} \cap B_{r}\left(y_{1}\right) \subset \mathcal{N}_{u}$. By the uniqueness for the Cauchy problem, $S_{1}$ contains at least one point $y_{1}^{\prime} \notin \mathcal{C}_{v}$. Thus, without loss of generality, we can assume that there exists a flat point $y_{1} \in \mathcal{N}_{u} \backslash \mathcal{C}_{v}$.

We arbitrarily fix a point $x_{1}$ belonging to the interior of one of the cells of $\partial G$. Again by the uniqueness for the Cauchy problem, we can assume, without loss of generality, that $\frac{\partial v}{\partial \nu} \neq 0, \nu$ being the interior unit normal to $\partial G$ at the point $x_{1}$.

We choose $\gamma$ according to Proposition [3.6. Then, applying iteratively Lemma 3.7. we can find a strictly increasing sequence $\left\{t_{n}\right\}_{n \in \mathbb{N}}$ such that, for any $n, y_{n}=$ $\gamma\left(t_{n}\right)$ is a flat point of $u$ and, by construction of $\gamma, y_{n}$ is not a critical point of $v$. Since $\mathcal{N}_{u}$ is bounded and $\lim _{t \rightarrow+\infty}\|\gamma(t)\|=+\infty$, there exists a finite $T$ such that $\lim _{n \rightarrow+\infty} t_{n}=T$. We have that $\tilde{y}=\gamma(T)$ belongs to $\mathcal{N}_{u}$ and, again by the properties of $\gamma, \tilde{y}$ is not a critical point of $v$ and $\gamma$ is orthogonal to $\mathcal{N}_{v}$ there. Therefore, there exists $\delta>0$ such that $v(\gamma(t)) \neq 0$ for every $T-\delta<t<T$ and this contradicts the fact that $\gamma\left(t_{n}\right) \in \mathcal{N}_{u}$ for any $n$.

\section{ACKNOWLEDGEMENT}

The authors wish to express their gratitude to Adrian Nachman and Masahiro Yamamoto for kindly sending their respective preprints 4 and 1]. The research reported in this paper originated at the 2003 Oberwolfach meeting "Inverse Problems in Wave Scattering and Impedance Tomography". The authors wish to thank the organizers Martin Hanke-Bourgeois, Andreas Kirsch and William Rundell, and the Mathematisches Forschungsinstitut Oberwolfach.

\section{REFERENCES}

1. J. Cheng and M. Yamamoto, Uniqueness in an inverse scattering problem within non-trapping polygonal obstacles with at most two incoming waves, Inverse Problems 19 (2003), pp. 13611384. MR2036535 (2004k:35394)

2. D. Colton and R. Kress, Inverse Acoustic and Electromagnetic Scattering Theory, SpringerVerlag, Berlin Heidelberg New York, 1998. MR.1635980|(99c:35181)

3. H. S. M. Coxeter, Regular Polytopes, Dover, New York, 1973. MR0370327 (51:6554)

4. C. Liu and A. Nachman, A scattering theory analogue of a theorem of Polya and an inverse obstacle problem, preprint (1994).

5. A. G. Ramm and A. Ruiz, Existence and uniqueness of scattering solutions in non-smooth domains, J. Math. Anal. Appl. 201 (1996), pp. 329-338. MR.1396903 (97b:35039)

Dipartimento di Matematica e Informatica, Università degli Studi di Trieste, Trieste, Italy

E-mail address: alessang@univ.trieste.it

Dipartimento di Matematica e Informatica, Università degli Studi di Trieste, Trieste, Italy

E-mail address: rondi@univ.trieste.it 